\title{
La Suisse au cœur
}

Chaque Nouvelle Année, les équipes rédactionnelles de médecine/sciences profitent de l'occasion pour souhaiter à tous leurs lecteurs un an de recherches fructueuses et de succès. Il n'est certainement pas dans notre esprit d'y manquer pour 1998 et si ces voux prennent, dans les paragraphes qui suivent, un tour un peu inhabituel c'est en espérant, déjà, qu'il ne s'agit que d'une exception et que, dès l'an prochain, nous pourrons réaffirmer sans réticence notre optimisme quant au développement de la recherche biomédicale et de ses retombées pour la médecine.

Notre regard se tourne toutefois avec une réelle appréhension, à l'aube de cette année 1998, vers la Suisse où un combat vital pour l'ensemble du monde scientifique est en train de se mener. Au cours de l'année qui vient, en effet - la date vient juste d'être fixée au 6juin 1998 - la population suisse va être appelée à décider, par la voie d'un référendum, tout simplement du sort de la recherche biologique dans le pays. Les «verts" suisses, appuyés à présent par le parti socialiste, ont en effet réussi à faire organiser une "votation» (un référendum d'initiative populaire) dont la conséquence - si elle est acceptée par une majorité des électeurs - serait d'interdire toute création d'organismes génétiquement modifiés, y compris des souris, mouches, vers et plantes... La Suisse, actuellement l'un des plus grands pays pour la recherche biomédicale aussi bien que pour l'industrie pharmaceutique, est ainsi menacée d'un recul scientifique et technique dont on ne peut sans doute évaluer les retombées qu'en regardant celles - encore sensibles 50 ans plus tard - du lyssenkisme en ex-Union Soviétique. médecine/ sciences est, bien évidemment, aux côtés des scientifiques suisses qui mènent un combat acharné contre cette terrible menace, et nos colonnes leur sont ouvertes autant qu'ils le jugeront utile. Comme l'écrivait récemment l'un des porte-parole les plus prestigieux des scientifiques suisses, Rolf Zinkernagel (Science, 14 no- vembre 1997), ce référendum pose à l'ensemble de la communauté scientifique la question de sa responsabilité face à la société. Il est inacceptable, et il doit être inconcevable, de légiférer sur la technologie scientifique comme le réclament, en Suisse, des groupes qui - après avoir par trois fois tenté d'interdire l'expérimentation animale - s'attaquent aujourd'hui à la technologie du gène, cour de la recherche biologique moderne. Réciproquement, on pourrait dire "en échange», la communauté scientifique doit avoir en permanence le souci de mettre ses résultats à la disposition du plus grand nombre et de contribuer aux débats provoqués par les perspectives qu'ouvrent ses recherches.

Le référendum "scientophobe» auquel se prépare la Suisse met sans doute en lumière l'insuffisance des moyens consacrés à l'information et à l'éducation scientifique, à l'école comme ailleurs. Le renforcement de ces moyens nécessite une chaîne d'élaboration et de transmission de la science dont tous les maillons sont importants, depuis les revues scientifiques primaires jusqu'aux journaux d'éveil pour les enfants, en passant par l'enseignement scolaire et universitaire et le journalisme scientifique. C'est dans cette chaîne de l'élaboration et de la transmission du savoir biologique et médical que médecine/sciences s'inscrit depuis douze ans, et notre but pour l'année à venir est de le faire toujours mieux, au service de notre communauté scientifique. Le rôle que nous nous sommes donné, depuis les origines, est d'établir avec précision et rigueur les bases du débat scientifique le plus pointu, pour le rendre intelligible à l'ensemble de la communauté scientifique et médicale et, ainsi, irriguer les champs de recherche de tous à partir des résultats de chacun. L'enseignement de la biologie et de la médecine est, évidemment, un des principaux bénéficiaires de cette recherche synthétique que traduisent les articles de médecine/sciences. Nous espérons ainsi contribuer, aussi fortement que nous le pouvons, à l'amélioration de la compréhension par tous des résultats de la science et des perspectives qu'ils impliquent, affaiblissant par là les groupes qui font leur lit de l'ignorance et de l'incompréhension.

En tant que scientifique-fondateur d'abord, membre du comité de rédaction ensuite, de rédacteur en chef français depuis 12 ans enfin, Axel Kahn a été un acteur essentiel dans l'élaboration du projet puis dans le développement de médecine/sciences. Comme nos lecteurs le liront dans l'éditorial qu'il signe dans ce numéro (page 4), Axel Kahn a été appelé à de hautes fonctions qui le mettent dans l'incapacité de maintenir l'investissement majeur qu'il consacrait à notre revue. Il a donc décidé de "passer la main " tout en continuant à assumer un rôle important dans le Comité de Direction de médecine/sciences qui est à présent mis en place. A son inspiration, les équipes rédactionnelles de médecine/sciences ont préparé au cours de ces derniers mois des modifications de leurs structures destinées à rendre le journal encore meilleur, dans sa couverture disciplinaire et dans sa liaison entre la biologie et la médecine. Nos lecteurs découvriront ainsi dans les mois qui viennent un nouveau Comité éditorial et un Comité scientifique largement remanié, avec l'espoir que, dès 1998, chacun pourra apprécier tout à la fois la continuité et le changement de médecine/sciences. Au moment où il cède la place de Rédacteur en chef qu'il a si brillamment occupée pendant douze ans, nous savons que nous nous faisons l'écho de tous les lecteurs de $m e ́-$ decine/sciences en remerciant Axel Kahn de l'œuvre considérable qu'il a accomplie dans ces fonctions et en lui souhaitant le même succès dans les tâches nouvelles qui lui sont confiées.

Bonne Année 1998 à tous !

Marc Peschanski
Rédacteur en chef, Paris
Michel Bergeron
Rédacteur en chef, Montréal

